\title{
High mass star formation in the infrared dark cloud G11.11-0.12
}

\author{
T. Pillai, F. Wyrowski, K. M. Menten, and E. Krügel
}

Max-Planck-Institut für Radioastronomie, Auf dem Hügel 69, 53121 Bonn, Germany

e-mail: thushara@mpifr-bonn.mpg.de

Received 8 October 2004 / Accepted 22 September 2005

\section{ABSTRACT}

We report detection of moderate to high-mass star formation in an infrared dark cloud (G11.11-0.12) where we discovered class II methanol and water maser emission at $6.7 \mathrm{GHz}$ and $22.2 \mathrm{GHz}$, respectively. We also observed the object in ammonia inversion transitions. Strong emission from the $(3,3)$ line indicates a hot $(60 \mathrm{~K})$ compact component associated with the maser emission. The line width of the hot component $\left(4 \mathrm{~km} \mathrm{~s}^{-1}\right)$, as well as the methanol maser detection, are indicative of high mass star formation. To further constrain the physical parameters of the source, we derived the spectral energy distribution (SED) of the dust continuum by analysing data from the 2MASS survey, HIRAS, MSX, the Spitzer Space Telescope, and interferometric $3 \mathrm{~mm}$ observations. The SED was modelled in a radiative transfer program: a) the stellar luminosity equals $\sim 1200 L_{\odot}$ corresponding to a ZAMS star of $8 M_{\odot}$; b) the bulk of the envelope has a temperature of $19 \mathrm{~K}$; c) the mass of the remnant protostellar cloud in an area $8 \times 10^{17} \mathrm{~cm}$ or $15^{\prime \prime}$ across amounts to $500 M_{\odot}$, if assuming standard dust of the diffuse medium, and to about $60 M_{\odot}$, should the grains be fluffy and have ice mantles; d) the corresponding visual extinction towards the star, $A_{\mathrm{V}}$, is a few hundred magnitudes. The near IR data can be explained by scattering from tenuous material above a hypothetical disk. The class II methanol maser lines are spread out in velocity over $11 \mathrm{~km} \mathrm{~s}^{-1}$. To explain the kinematics of the masing spots, we propose that they are located in a Kepler disk at a distance of about $250 \mathrm{AU}$. The dust temperatures there are around $150 \mathrm{~K}$, high enough to evaporate methanol-containing ice mantles.

Key words. masers - stars: formation - molecular data - radiative transfer - astronomical databases: miscellaneous

\section{Introduction}

Infrared dark clouds (IRDCs) are cold, dense molecular clouds seen silhouetted against the bright diffuse mid-infrared emission of the Galactic plane. They were discovered during mid-infrared imaging surveys with the Infrared Space Observatory (ISO) (Perault et al. 1996) and the Mid-course Space Experiment $\left(\mathrm{MSX}^{1}\right)$ (Egan et al. 1998). The mm/submm observations revealed that typical IRDCs have gas densities of $n>10^{6} \mathrm{~cm}^{-3}$, temperatures of $T<20 \mathrm{~K}$, and sizes of 1-10 pc (Carey et al. 1998). To determine the mass distribution toward the IRDCs, Carey et al. (2000) obtained images of the 450 and $850 \mu \mathrm{m}$ emission using SCUBA. All observed IRDCs contain 1-4 bright sub-millimeter emission peaks ( $>1 \mathrm{Jy} /$ beam at $850 \mu \mathrm{m}$ ) surrounded by an envelope of emission, which matches the morphology of the IRDC in midinfrared extinction.

Carey et al. (2000) suggest that IRDCs might be sites of early phases of high-mass star formation, however studies of their star formation content are still rare. There are,

\footnotetext{
1 This research made use of data products from the Midcourse Space Experiment. Processing of the data was funded by the Ballistic Missile Defense Organization with additional support from the NASA Office of Space Science. This research also made use of the NASA/ IPAC Infrared Science Archive, which is operated by the Jet Propulsion Laboratory, California Institute of Technology, under contract with the National Aeronautics and Space Administration.
}

indeed two cases where detailed studies were done towards the IRDCs, although the original object identification was not based on the extinction in the MIR. Based on the submm dust continuum and line observations, Sandell (2000) conclude that NGC $6334 \mathrm{I}(\mathrm{N})$ is a high-mass Class 0 object. Similarly, Garay et al. (2002) suggest that IRAS 16272 is a dense massive core in a very early evolutionary stage, distinguished by being luminous without any associated Ultracompact HII regions (UCHIIR). Toward the IRDC G79.3+0.3 P1, Redman et al. (2003) reported associated low and intermediate mass YSOs. Also, Teyssier et al. (2002) found young embedded stars associated with the IRDCs they studied, but do not give further details on them. Observations of IRDC G11.11-0.12 are discussed by Johnstone et al. (2003) and, although they noticed signs of star formation associated with their SCUBA source P1, they did not find evidence of massive star formation. Identifying the earliest stages in the formation of massive stars is currently a crucial step in our understanding of massive star formation, so we started a project to search for methanol masers toward cold massive cores.

Class II methanol $\left(\mathrm{CH}_{3} \mathrm{OH}\right)$ masers (CIIMMs) are almost always found in high-mass star-forming regions (HMSFRs). It turned out that not all of the CIIMM are associated with UCHIIR (Walsh et al. 1998). Based on high spatial-resolution radio continuum and $6.67 \mathrm{GHz}$ methanol spectral-line data in 364 sources towards IRAS-selected regions, Walsh et al. (1998) 
show that the methanol maser is most likely present before an observable UCHIIR is formed around a massive star and is quickly destroyed as the UCHIIR evolves. However, this is disputed by Phillips et al. (1998) who argue that the non detection of radio continuum emission associated with methanol masers is because of the association of the maser sites with low/intermediate mass stars which do not ionise their immediate surroundings. To date no CIIMM has ever been detected toward a low-mass SFR, with the possible exception of NGC 2024-FIR4 (Minier et al. 2003), which, by the way, is the only known CIIMM in the whole Orion Giant Molecular Cloud (GMC) complex. Walsh et al. (2001) in another study of 31 methanol maser sites and 19 UCHIIR in Mid-infrared ( 10.5 and $20 \mu \mathrm{m}$ ) find that the observations are consistent with the maser emission being powered by the MIR source. Walsh et al. (2001) also find strong evidence of infall/outflow in different molecular tracer. Beuther et al. (2002) find a mean separation of $\sim 0.19 \mathrm{pc}$ between the $\mathrm{CH}_{3} \mathrm{OH}$ and $\mathrm{cm}$ emission for their sample of 29 massive star forming regions. Subsequently, in a continuum mapping reported by Walsh et al. (2003) toward $716.7 \mathrm{GHz}$ maser fields, they find that most methanol masers are within $10^{\prime \prime}$ of a sub-mm peak. Furthermore, a recent $1.2 \mathrm{~mm}$ continuum survey in massive star forming regions with methanol maser sites and/or radio continuum emission, reported by Hill et al. (2005) shows that these tracers of massive star formation are associated with heavily embedded protostars. These arguments taken together with the non detection of radio continuum make a convincing case of CIIMMs signposting the earliest stages of high mass star formation.

Here we report the discovery of CIIMM emission toward the IRDC G11.11-0.12 and discuss the properties of the protostar heavily embedded in the IRDC detected with SPITZER in the MIR. In Sect. 2, we describe our observation in various molecular tracers with the Effelsberg 100-m telescope, Australian Telescope Compact Array, Very Large Array and the Berkeley-Illinois-Maryland-Association interferometer. In Sect. 3 we present the evidences for active star formation in the IRDC and model the ammonia emission. The discussion on the Spectral Energy Distribution (SED) of the embedded source and modelling in spherical symmetry follows through in Sect. 4. The observed properties of the CIIMM are entirely comparable with the properties of numerous other such sources detected in high-mass star forming regions. Therefore, its presence makes G11.11-0.12, an IRDC in which active high-mass star formation has been unequivocally established.

\section{Observations}

\section{1. $\mathrm{CH}_{3} \mathrm{OH}$ maser}

We observed the class II methanol maser at $6.7 \mathrm{GHz}\left(5_{1}-6_{0} \mathrm{~A}^{+}\right.$ transition) toward the submm peak IRDC G11.11 P1 $\left(\alpha_{\mathrm{J} 2000}=\right.$ 18:10:28.402, $\left.\delta_{\mathrm{J} 2000}=-19: 22: 29.00\right)$ with the Effelsberg $100-\mathrm{m}$ telescope $\mathrm{e}^{2}$ on 15 June 2003 . The frontend was the facility $5 \mathrm{~cm}$ receiver tuned to $6668.518 \mathrm{MHz}$. We used the

\footnotetext{
${ }^{2}$ Based on observations with the 100-m telescope of the MPIfR (Max-Planck-Institut für Radioastronomie) at Effelsberg.
}

8192 channel auto-correlator with 2 subunits of $10 \mathrm{MHz}$ bandwidth to be able to reach a spectral resolution of $\approx 0.25 \mathrm{~km} \mathrm{~s}^{-1}$. The beam width of $122^{\prime \prime}$ was determined from drift scans over compact continuum sources, which also served to check the pointing. The observations were repeated with the $100-\mathrm{m}$ telescope on 5 August 2004.

On 11 January 2004, we followed up the observations of the 6.7 GHz Class II $\mathrm{CH}_{3} \mathrm{OH}$ maser with the Australian Telescope Compact Array (ATCA) in the FULL_4_1024-128 correlator configuration, employing the snapshot mode with a series of 6 short integration cuts of $5 \mathrm{~min}$. Since ATCA can observe two frequencies simultaneously, we chose the $8.64 \mathrm{GHz}$ continuum band covering a bandwidth of $128 \mathrm{MHz}$ frequencies. The $1 \sigma$ sensitivity achieved is $\sim 0.2 \mathrm{mJy} /$ beam. Phase calibration was done using B1817-254. The primary beam of ATCA at this frequency is $\sim 8^{\prime}$, and the effective $H P B W$ of the synthesized beam equals $2.03^{\prime \prime} \times 6.24^{\prime \prime}$. The spectral resolution was $0.2 \mathrm{~km} \mathrm{~s}^{-1}$, and the $1 \sigma$ sensitivity $0.5 \mathrm{Jy}$. The ATCA spectrum is essentially identical to the 2003 Effelsberg spectrum.

\subsection{Ammonia observation}

The maser detection prompted us to search for the $\mathrm{NH}_{3}(3,3)$ and $(4,4)$ transitions with the Effelsberg $100-\mathrm{m}$ telescope in April 2004. With the AK 8192 backend, we were able to observe the $(1,1),(2,2),(3,3)$, and $(4,4)$ transitions in both polarisations simultaneously using the $K$-band receiver. With 8 subunits of $10 \mathrm{MHz}$ bandwidth, the resulting spectral resolution was $\approx 0.2 \mathrm{~km} \mathrm{~s}^{-1}$ after smoothing the data to improve the signalto-noise ratio. The beam at the $\mathrm{NH}_{3}$ frequencies was $40^{\prime \prime}$. The observations were performed in the frequency switching mode. Pointing was checked at hourly intervals by continuum scans on G10.62. We estimate, the pointing to be accurate within $6^{\prime \prime}$, with the pointing scans used for the absolute calibration.

\section{3. $\mathrm{VLA} \mathrm{H}_{2} \mathrm{O}$ maser observation}

The $22.2 \mathrm{GHz} \mathrm{H}_{2} \mathrm{O}$ maser observations were done with the Very Large Array (VLA) on 24 August 2004 in its D configuration and in 2 polarisations with a spectral resolution of $0.33 \mathrm{~km} \mathrm{~s}^{-1}$, a synthesized $H P B W$ of $\sim 0.4^{\prime \prime}$, and a primary beam of $\sim 120^{\prime \prime}$. The standard interferometer mode was used with a total integration time of $20 \mathrm{~min}$ on source, split into sessions. Phase calibration was done using J1733-130, and the flux calibrator was 3C 286.

\subsection{BIMA observation}

The $3 \mathrm{~mm}$ observations were carried out from September 1999 to July 2000 with the Berkeley-Illinois-Maryland-Association interferometer (BIMA) in its B, C, and D configuration sampling spatial structures from $3^{\prime \prime}$ to $60^{\prime \prime}$. Continuum images were obtained with a combination of 86 and $93 \mathrm{GHz}$ tracks centred with a beam size of $8.3^{\prime \prime} \times 3.9^{\prime \prime}$ reaching an rms of $1.6 \mathrm{mJy}$. The total bandwidth of both sidebands was $\sim 900 \mathrm{MHz}$. 


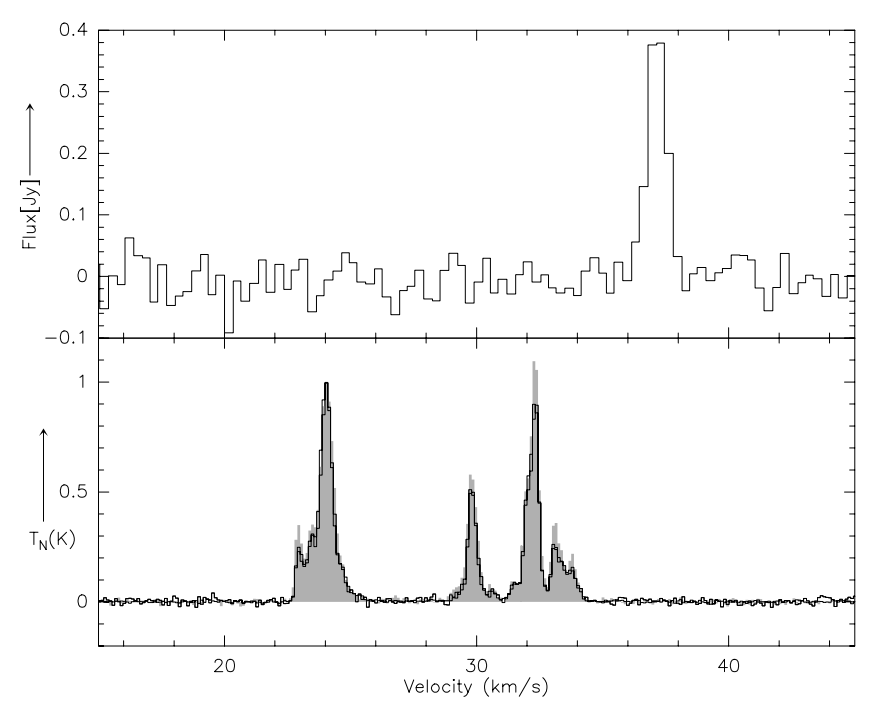

Fig. 1. Lower panel: Effelsberg 100-m spectra of the $\mathrm{CH}_{3} \mathrm{OH}$ emission at $6.7 \mathrm{GHz}$. The filled spectrum corresponds to June 2003 and the solid lines to August 2004 observations. The blue shifted component is normalised to unity in both cases. Upper panel: $22.2 \mathrm{GHz}$ water maser spectra obtained with the VLA.

\section{Results}

\section{1. $\mathrm{CH}_{3} \mathrm{OH}$ maser}

The solid line in Fig. 1 displays the $6.7 \mathrm{GHz}$ methanol maser spectrum toward G11.11P1 detected in August 2004 with the Effelsberg 100-m telescope, while the filled region shows the June 2003 data. As the Effelsberg 100-m absolute calibration has an uncertainty of $\sim 20 \%$, the observations taken with two different instruments and at different times have been normalised with respect to one of the features, so that absolute calibration is no longer an issue. The maser features cover a velocity range of about $11 \mathrm{~km} \mathrm{~s}^{-1}$, with the peaks at $32 \mathrm{~km} \mathrm{~s}^{-1}$ in June 2003 and at $24 \mathrm{~km} \mathrm{~s}^{-1}$ in August 2004. Thus there is clear evidence of a variation in the relative line intensities of about $10 \%$. From mapping the source, we find that the masing spots originate at the position $\mathrm{P} 1$ from an area that is pointlike with respect to the Effelsberg beam. There is no evidence of another maser source. The follow-up observations obtained six months later with ATCA reveal no obvious changes in the spectrum and also indicate that the maser originates from a single core. The peak flux of the brightest maser feature from the ATCA observations is $22 \mathrm{Jy}$.

The position of the integrated maser emission is plotted in Fig. 2. There, the left panel shows the MSX map of the filamentary dark cloud overlaid on the SCUBA submm map from Carey et al. (2000), and the right panel is a blow-up of the SCUBA image around P1, together with contours of 2MASS $K_{\mathrm{s}}{ }^{3}$ band and the $3 \mathrm{~mm}$ continuum emission. To estimate the positional accuracy of the maser observations, we split the phase calibrator observations into two sets and

\footnotetext{
3 The atlas image obtained is part of the Two Micron All Sky Survey (2MASS), a joint project of the University of Massachusetts and the Infrared Processing and Analysis Center/California Institute of Technology, funded by the NASA and the NSF.
}

cross-calibrate the second set with a self-calibrated first set, which results in a position accuracy of $\left(0.05^{\prime \prime},-0.15^{\prime \prime}\right)$. This is a lower limit, but since our source is less than $10^{\circ}$ away from the calibrator, baseline errors are not likely to add to the error. To cross-check this, we reduced other observations towards an already known maser source and found a positional accuracy of $\left(0.105^{\prime \prime},-0.75^{\prime \prime}\right)$. ATCA is an east west array, so the beamwidth in declination is greater by a factor cosec (Dec); and for those sources with declination north of $-24 \mathrm{deg}$, complete $u-v$ coverage is unobtainable.

When the sub-spots are analysed in position-velocity plots, they fall nicely along an arc, as predicted for a disk (Minier et al. 1998; Norris et al. 1998). There is also a linear velocity trend. This is shown in Fig. 3 where a point source model was fitted to individual velocity channels. The one sigma position uncertainty is proportional to the SNR (Reid et al. 1988); hence, with a SNR 15-30 for the different velocity features, we reach a sub-arcsecond accuracy. The relatively weak peak at $34 \mathrm{~km} \mathrm{~s}^{-1}$ was not included due to its large positional uncertainty.

\section{2. $3 \mathrm{~mm}$ continuum emission}

The BIMA image shows a compact, slightly resolved $3 \mathrm{~mm}$ continuum source that coincides with the SCUBA sub-mm peak shown in Fig. 2. A 2-D Gaussian fit to the emission yields a source size of $0.25 \times 0.12 \mathrm{pc}$ with a PA $3^{\circ}$. The peak flux of $\sim 12 \mathrm{mJy}$ corresponds to a brightness temperature of $\sim 28 \mathrm{mK}$. Our cm continuum observations with ATCA at $8.4 \mathrm{GHz}$, along with searches in Galaxy-wide $\mathrm{cm}$ continuum surveys, reveal no detectable free-free emission. In order to check whether one expects to find free-free emission at $3 \mathrm{~mm}$, we extrapolated the ATCA upper limit to that at $3 \mathrm{~mm}$, assuming optically thick emission. We estimated a mm flux of $25.3 \mathrm{mJy}$ relative to the ATCA upper limit of $\sim 0.2 \mathrm{mJy}$. Thus the contribution from free-free emission cannot be ruled out. For a typical value of $10000 \mathrm{~K}$ for the ionisation temperature from an optically thick ionised gas cloud, we get an emission measure $E M>4 \times 10^{10} \mathrm{pc} \mathrm{cm}^{-3}$ at $90 \mathrm{GHz}$, a very high value that is more suitably assigned to Hypercompact HII regions (Kurtz 2002). Such a region with typical sizes $<0.05$ pc would have been point-like with respect to the BIMA beam, while we found that the source is partially resolved. Thus, the $3 \mathrm{~mm}$ emission is most likely due to dust.

\section{3. $\mathrm{H}_{2} \mathrm{O}$ maser}

The position of the $\mathrm{H}_{2} \mathrm{O}$ maser appears offset from the $\mathrm{CH}_{3} \mathrm{OH}$ maser position, as well as from the BIMA $3 \mathrm{~mm}$ continuum peak, within the respective positional errors. This might indicate that, although both $\mathrm{CH}_{3} \mathrm{OH}$ and $\mathrm{H}_{2} \mathrm{O}$ masers occur in warm and dense environments, they may not spatially coincide due to different excitation mechanisms (Beuther et al. 2002).

Water masers can be explained by collisional pumping with $\mathrm{H}_{2}$ molecules in shocks associated with outflows (Elitzur et al. 1989). Alternatively, the pumping can occur by accretion shocks in disks (Garay \& Lizano 1999). The maser is weak 

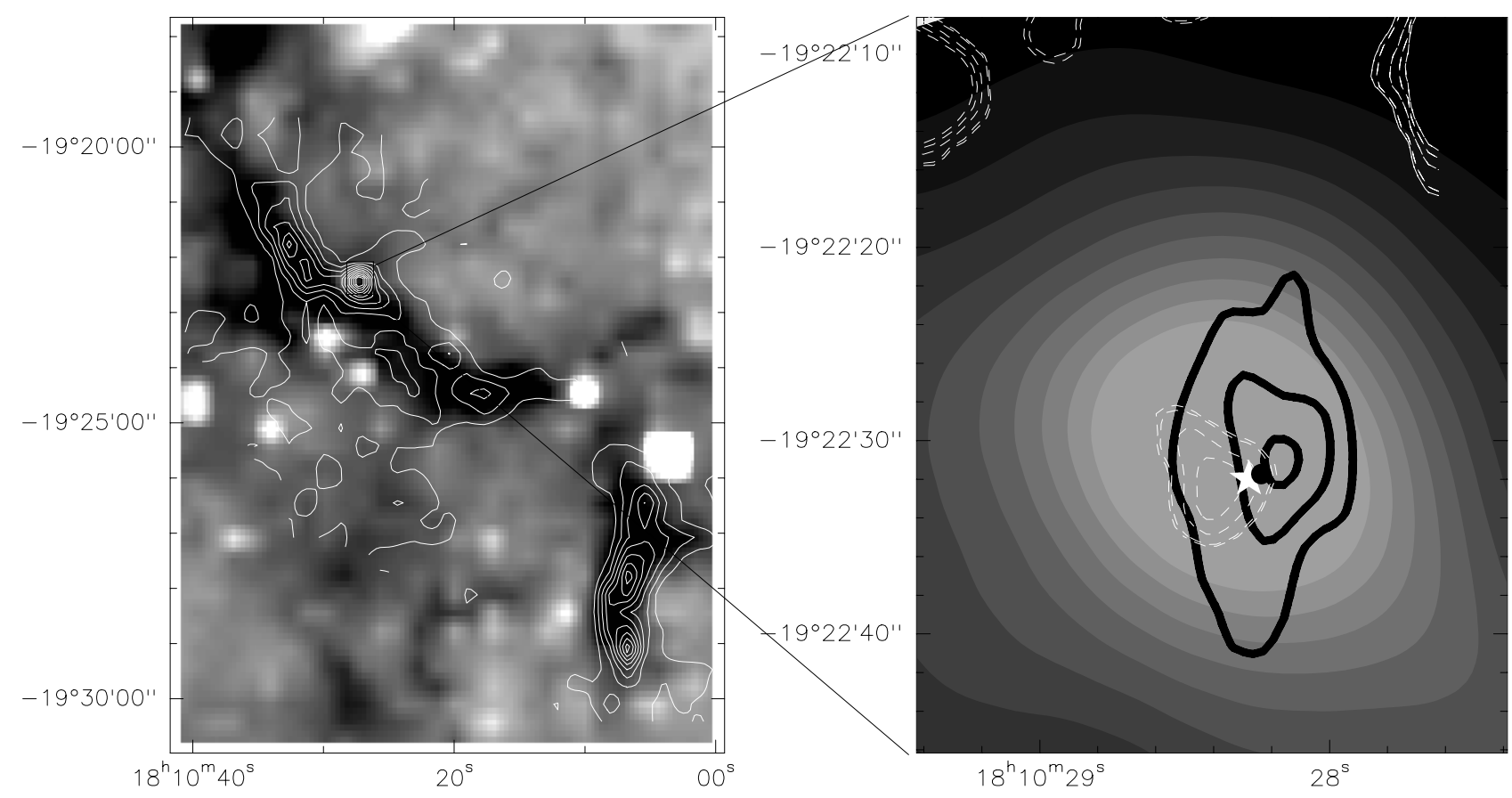

Fig. 2. Left: the $8 \mu \mathrm{m}$ image of G11.11 with SCUBA $850 \mu \mathrm{m}$ (Carey et al. 2000) overlay. Right: BIMA 3 mm continuum image towards P1 in contours, with contour level $\sim 1.6 \mathrm{mJy}(-3,3,5,7)$ and $2 \mathrm{MASS} K_{\mathrm{s}}$ band in dashed contours on the $850 \mu \mathrm{m}$ image. The star denotes the $\mathrm{H}_{2} \mathrm{O}$ maser and the filled circle the $\mathrm{CH}_{3} \mathrm{OH}$ maser position.

$(\sim 0.3 \mathrm{Jy})$ with a feature offset of $\sim 7 \mathrm{~km} \mathrm{~s}^{-1}$ from the systemic velocity as shown in the upper panel of Fig. 1. This agrees well with the picture of water masers being variable and having large velocity spread.

The maser spot is not spatially resolved over its velocity structure. We performed a model fit to the individual velocity channels over the single feature. Unlike the $\mathrm{CH}_{3} \mathrm{OH}$ masers, the $\mathrm{H}_{2} \mathrm{O}$ maser spots do not show any systematic gradient. Since the velocity structure is also found to be spread on a much higher angular scale than the $\mathrm{CH}_{3} \mathrm{OH}$ maser, it may be tracing the outflow. Higher angular resolution is needed to reveal structures that could be either a shock front of the high-velocity outflow very close to the central object or part of the accretion disk itself. We also serendipitously discovered another water maser that was offset by more than $1^{\prime}$. The feature is brighter and situated at the systemic velocity of the source. The absolute position of the maser peak is at $\alpha_{\mathrm{J} 2000}=18: 10: 33.58$, $\delta_{\mathrm{J} 2000}=-19: 22: 50.3$.

\subsection{Ammonia towards G11.11P1}

Figure 4 shows the ammonia lines observed with the 100-m telescope toward G11.11P1, and the line parameters are given in Table 1 . For the $(4,4)$ line that was not detected, the $1 \sigma \mathrm{rms}$ noise in the spectrum is given instead.

The line widths from Gaussian fits when taking the hyperfine satellites for the $(1,1)$ and $(2,2)$ spectra into account increases with the excitation of the lines. The $(3,3)$ transition even shows, a broad wing besides the line core. From a Boltzmann plot of the ammonia emission, we conclude that the ammonia emission cannot be explained with a single temperature. From just the $\mathrm{NH}_{3}(1,1)$ and $(2,2)$ data, we got a rotational

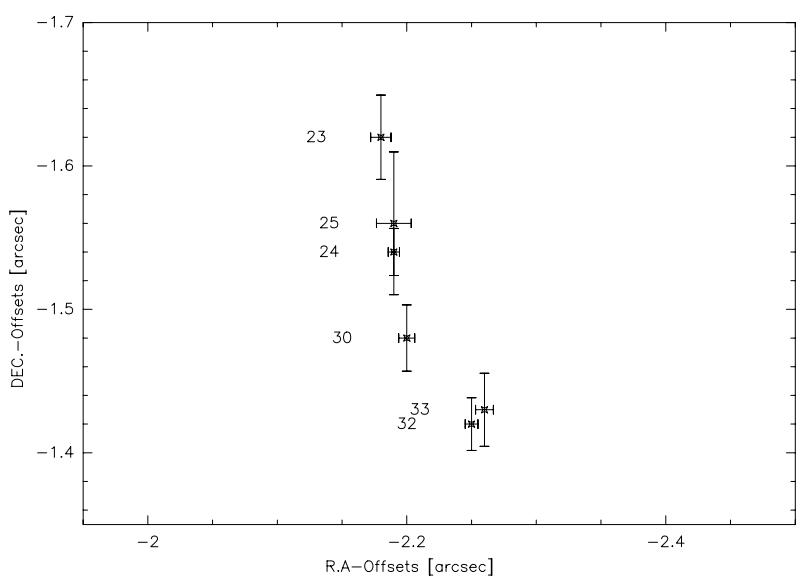

Fig. 3. Velocity structure of the $\mathrm{CH}_{3} \mathrm{OH}$ maser emission. Each data point gives the position of the model fits to the individual velocity channels. The corresponding $V_{\mathrm{LSR}}$ in $\mathrm{km} \mathrm{s}^{-1}$ is also shown.

Table 1. Effelsberg 100-m line parameters.

\begin{tabular}{lrcc}
\hline \hline Transition & $\begin{array}{r}v_{\text {LSR }} \\
\left(\mathrm{km} \mathrm{s}^{-1}\right)\end{array}$ & $\begin{array}{c}T_{\text {MB }} \\
(\mathrm{K})\end{array}$ & $\begin{array}{c}F W H M \\
\left(\mathrm{~km} \mathrm{~s}^{-1}\right)\end{array}$ \\
\hline $\mathrm{NH}_{3} 1-1$ & $29.78(0.01)$ & $4.1(0.4)$ & $1.29(0.02)$ \\
$\mathrm{NH}_{3} 2-2$ & $29.78(0.02)$ & $1.58(0.07)$ & $1.81(0.06)$ \\
$\mathrm{NH}_{3} 3-3$ & $29.77(0.07)$ & $0.64(0.05)$ & $3.22(0.21)$ \\
$\mathrm{NH}_{3} 4-4$ & & $(0.065)$ & \\
\hline
\end{tabular}

temperature of $\sim 14 \mathrm{~K}$. The rotational temperature derived from the $(2,2)$ and $(3,3)$ lines was estimated to be $\sim 30 \mathrm{~K}$.

This evidence of two temperature components prompted us to fit the spectrum with two components simultaneously using 


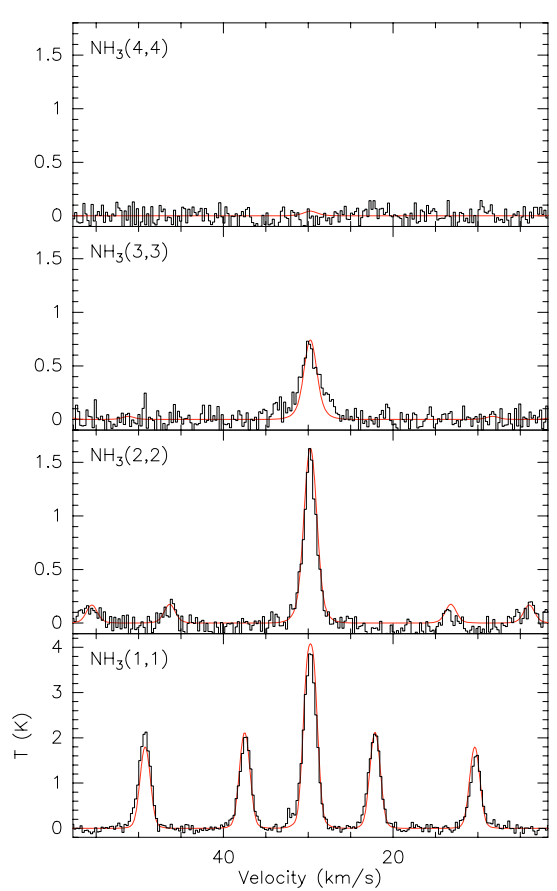

Fig. 4. Top to bottom: spectra of the $\mathrm{NH}_{3}(J, K)=(1,1),(2,2),(3,3)$, and $(4,4)$ transitions. All spectra were taken towards the submm peak position given in the text. The fit discussed in Sect. 3.4 is overlaid.

XCLASS (Schilke, P., private communication). The program then yields the rotation temperature, column density and source size for a given telescope size assuming a Gaussian structure, and produces a synthetic spectrum in agreement with observations. Table 2 lists the fit results. Thus the broader component appears to be much hotter $(60 \mathrm{~K})$ and is very compact $\left(3.3^{\prime \prime}\right)$. Actually, the fit is not unique since there is degeneracy between column density and the source size. But a large source size would mean a still higher luminosity, which would put the source in the spectral type O9. It is highly unlikely that an HII region around such a star goes undetected. On the other hand, a much smaller source size with very high column density would mean extremely dense gas with critical density high enough to excite the $(4,4)$ level, which we do not detect.

Additionally, we fit the ammonia spectra with a spherical symmetric model (Wyrowski et al. 2003) using both a core component with a temperature power law, consistent with internal heating from a $1200 L_{\odot}$ embedded source, and a more extended, cooler $(10 \mathrm{~K})$ lower density clump. For the core component, the luminosity, and the outer and inner radii were taken from the dust continuum modelling, which will be presented in Sect. 4.3.1. Assuming an ammonia abundance of $10^{-8}$, the density in the core is $7 \times 10^{5} \mathrm{~cm}^{-3}$, which is consistent with the modelling in Sect. 4.3.1, and is a factor 10 lower in the cooler clump with a size of $40^{\prime \prime}$. For the core component, a line width gradient was modelled with $4 \mathrm{~km} \mathrm{~s}^{-1}$ in the inner 2 arcsec and then decreasing to the clump component with $1.2 \mathrm{~km} \mathrm{~s}^{-1}$. The increasing line widths/turbulence towards the centre of the core is another indication of an embedded, massive young stellar object that is stirring up its environment, as well as the remaining line wings of the $(3,3)$ line, which indicates an additional outflow component.
Table 2. Model fit results.

\begin{tabular}{lrccc}
\hline \hline Component & $\begin{array}{r}T_{\text {rot }} \\
(\mathrm{K})\end{array}$ & $\begin{array}{c}\text { size } \\
\left({ }^{\prime \prime}\right)\end{array}$ & $\begin{array}{c}N_{\mathrm{NH}_{3}} \\
\left(10^{15} \mathrm{~cm}^{-2}\right)\end{array}$ & $\begin{array}{c}F W H M \\
\left(\mathrm{~km} \mathrm{~s}^{-1}\right)\end{array}$ \\
\hline $\mathrm{NH}_{3}$ cold & 15.4 & 23 & 1.8 & 1.34 \\
$\mathrm{NH}_{3}$ hot & 60 & 3.3 & 13.7 & 4 \\
\hline
\end{tabular}

\subsection{Archival data}

We collected the data from 4 galactic sky survey archives, namely 2MASS, Spitzer, MSX, and IRAS. The 2MASS point source catalog contains the $J H K$ fluxes for 3 point sources all within the 14" of the SCUBA beam. The Spitzer MIR data collected from the Spitzer data archive is part of the GLIMPSE Legacy survey in 4 of the Infrared Array Camera (IRAC) bands from $3.6-8 \mu \mathrm{m}$. We found 3 point sources in the $3.6 \mu \mathrm{m}$ band, while in the higher wavelength bands we found a single source that were coincident with the MSX $8 \mu \mathrm{m}$ point source. The FIR emission obtained from the high resolution IRAS data is not point like and might suffer from background emission. But IRAS point sources generally do not appear circular, primarily because the detector aperture were rectangular. We adopted an flux uncertainty of $20 \%$ in all cases as given by the respective Space centres.

\section{Discussion}

\section{1. $\mathrm{CH}_{3} \mathrm{OH}$ maser disk scenario}

Figure 1 contains 3 major peaks that is very reminiscent of a classical maser triplet, which is usually ascribed to a maser amplification from a disk in Keplerian rotation viewed edge on (Elmegreen \& Morris 1979).

According to Cesaroni (1990) and Ponomarev et al. (1994), the intensity ratio of the central to the red and blue peaks is determined by the width of the ring in which the conditions for line amplification are favourable, and the central peak vanishes when the ring becomes very small. The red and blue components of our spectrum are not equidistant to the central component that marks the systemic velocity (see also Table 2 of $\mathrm{NH}_{3}$ velocities). Such a distortion may be due to a tilt of the disk, in which case the physical parameters (density, temperature, and velocity) along the line of sight depend not only on the radial distance, but also on the height above the disk.

In principle, equidistant triplets can also arise from an expanding shell. We favour the disk scenario for several reasons. a) Variations in the line ratios, as argued by Cesaroni (1990), are readily explained for disks by radiative interaction between masing spots, whereas such an interaction would not be possible for an expanding shell because of opposite velocities of the masing. b) All 3 major peaks contain sub-peaks, which we interpret as arising from irregularities associated with the tilt or warp of the disk. c) The near-infrared emission and the Spitzer data discussed in Sect. 4.1.2 also seem to require a disk structure. 


\subsection{Mass and luminosity estimates}

The kinematic distance to G11.11 derived as $3.6 \mathrm{kpc}$ using the rotation curve of Clemens (1985) and the IAU standard values of $R_{0}=8.5 \mathrm{kpc}$ and $V_{0}=220 \mathrm{~km} \mathrm{~s}^{-1}$ by Carey et al. (1998) is $3.6 \mathrm{kpc}$. Carey et al. (2000) quote a gas mass of $67 M_{\odot}$ from their dust continuum observations at 850 micron. The virial masses estimated from ammonia observations range from 150-240 $M_{\odot}$ for the hot and the cold components within the beam $\left(40^{\prime \prime}\right)$ of Effelsberg. Similarly, by assuming the fractional abundance of $\mathrm{NH}_{3}$ (relative to $H_{2}$ ) to be on the order of $10^{-8}$, the $\mathrm{NH}_{3}$ column density translates to masses of 200-1600 $M_{\odot}$.

The CIIMM need elevated temperatures for two reasons: first, high methanol abundances are required to produce an adequate maser gain, and second, high temperatures are needed to produce pump photons. To effectively evaporate methanol off icy grain mantles, temperatures in excess of $100 \mathrm{~K}$ are needed (Sandford \& Allamandola 1993). Recent models of CIIMM pumping require gas temperatures $\sim 100 \mathrm{~K}$, somewhat higher dust temperatures, and densities of about $10^{7} \mathrm{~cm}^{-3}$. Therefore, the compact $\mathrm{CH}_{3} \mathrm{OH}$ maser emission we observe must arise from a hot, dense source. We may adopt the spread of the maser components, $0.25^{\prime \prime}$ or $900 \mathrm{AU}$, as the size of that source, which is comparable to the sizes of numerous other CIIMM regions. The Stefan-Boltzmann law delivers with a luminosity of $830 L_{\odot}$ for a source of the corresponding area and $T=100 \mathrm{~K}$. To fit all observed ammonia lines simultaneously, we presented a model in Sect. 3.4 that assumes an optically thin spherical dust cloud illuminated by a central point source, which then results in a radial temperature distribution of $T \propto r^{-0.4}$ (Wilner et al. 1995). With a luminosity of $1200 L_{\odot}$, we could explain the observed warm ammonia core embedded within a larger cold clump. In the model, the temperature reaches a temperature of $240 \mathrm{~K}$ at the inner radius, hence large enough to excite the methanol maser observed.

\subsection{SED}

\subsubsection{MIR and longer wavelengths}

The SED of G11.11 P1 shown in Fig. 5 contains fluxes due to dust emission at (i) $3 \mathrm{~mm}$ (BIMA, $8^{\prime \prime} \times 4^{\prime \prime}$ beam), (ii) at 450 and $850 \mu \mathrm{m}$ (JCMT), (iii) IRAS data, (iv) MSX upper limits at 12 and $21 \mu \mathrm{m}$, (v) an MSX detection at $8.2 \mu \mathrm{m}$, (vi) four detections with the Spitzer Space Telescope (at 3.6, 4.5, 5.8 and $8.0 \mu \mathrm{m}$ ), and (vii) three NIR data points from 2MASS.

Although for massive young stars, a time sequence from class 0 to class 3 has not been established, a high-mass-star equivalent to a class 0 object should also emit more than half a percent of its energy at submm wavelengths (André et al. 1993). For G11.11, the luminosity at $\lambda>300 \mu \mathrm{m}$ amounts to almost $3 \%$ of the total, so in this respect it could be considered an object equivalent to a Class 0 source. Similar arguments of a Class 0 equivalent object in high-mass-star-forming region have also been proposed by Motte et al. (2003) in the submm protoclusters in W43. They derive a submillimeter to bolometric luminosity ratio of $1.5-3 \%$ in W43-MM1 and MM2.
To estimate how the dust temperature and density vary within the envelope, we tried to reproduce the continuum emission in a self-consistent way by calculating the radiative transfer in spherical symmetry. However, when including the NIR fluxes, as well as the recently published data from the Spitzer archive, the assumption of strict radial symmetry had to be abandoned. We sketch below a possible, or even likely, structure of the source; but to confirmat its correctness we need information with higher spatial resolution, a difficult requirement for such a distant source. The model therefore remains rather qualitative.

Nevertheless, the observations yield a number of important constraints. a) The total luminosity is determined by the IRAS fluxes. While the $100 \mu \mathrm{m}$ flux may contain a fair contribution by diffuse emission (from cloud heating by the interstellar radiation field or scattered stellar sources), the $60 \mu \mathrm{m}$ flux indicating warm dust comes from a compact source with a luminosity of $\left.\sim 10^{3} L_{\odot} . b\right)$ As the source is only marginally resolved at $3 \mathrm{~mm}$, its outer cloud radius $r_{\text {out }}$ has to be smaller than $4 \times$ $\left.10^{17} \mathrm{~cm} . c\right)$ The submm $/ \mathrm{mm}$ fluxes lead to a total mass of several hundred $M_{\odot}$ when adopting standard interstellar dust with an absorption coefficient at $1 \mathrm{~mm}$ of $\sim 4 \times 10^{-3} \mathrm{~cm}^{2}$ per gram of interstellar matter (Krügel 2003). However, as this emission is optically thin, a substantial part may not be directly linked to the star, but may come from the background within the IRDC. d) The visual extinction to the star depends on how the dust is distributed. It is about $400 \mathrm{mag}$ if all the mass is in a sphere with constant density around the star, and even higher for a centrally condensed envelope, and less than $400 \mathrm{mag}$ in case of an additional background cloud. For comparison, we derive a column density $A_{\mathrm{V}}>60$ mag from the peak of the ammonia emission in a $40^{\prime \prime}$ beam. This column density is probably two or three times larger, depending on the compactness of the source. We then assumed conversion factors $\left[\mathrm{NH}_{3}\right] /\left[\mathrm{H}_{2}\right]=10^{-8}$ and $N_{\mathrm{H}}=2 \times 10^{21} A_{\mathrm{V}} \mathrm{cm}^{-2}$ (Motte et al. 1998).

The solid line in Fig. 5, which is compatible with the observations between $10 \mu \mathrm{m}$ and $3 \mathrm{~mm}$, comes from a spherical radiative transfer model (see Appendix A) with a central star of $L=1200 L_{\odot}$ and $15000 \mathrm{~K}$ surface temperature that is surrounded by a envelope whose inner and outer radii are $10^{15}$ and $3.5 \times 10^{17} \mathrm{~cm}$, respectively. The exact number for $T_{\text {eff }}$ is irrelevant, because the stellar radiation gets immediately absorbed. The dust density in the envelope is constant $\left(\rho_{\text {dust }}=3 \times 10^{-20} \mathrm{~g} \mathrm{~cm}^{-3}\right)$ implying an optical extinction $A_{\mathrm{V}} \simeq 400$ mag. It may, at most, be two times less, otherwise the model spectrum would not comply with the $21 \mu \mathrm{m}$ upper limit. If it is less than $400 \mathrm{mag}$, one has to invoke the above mentioned background cloud to explain the sub-mm fluxes. These model results are also consistent with our fits to the ammonia lines described in Sect. 3.4.

It is important to note that the model spectrum for the FIR does not rise again at shorter wavelengths and cannot account for the $8.2 \mu \mathrm{m}$ MSX and the Spitzer fluxes. Here an independent source is required. We propose that besides the star, there is a second blackbody emitter close to it. Its temperature is approximately $T=550 \mathrm{~K}$, its total luminosity $150 L_{\odot}$, and it suffers 150 mag of visual extinction. This is below the minimum estimate for $A_{\mathrm{V}}$ of the envelope, but the latter may, of course, be 


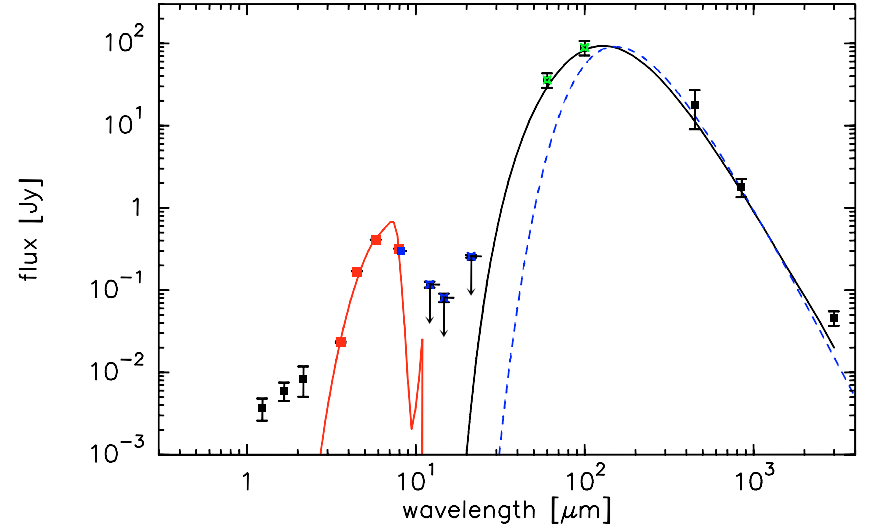

Fig. 5. The Spectral Energy Distribution of G11.11-0.12 P1. Squares with errorbars indicate observations, where two almost coincide at 8 and $8.2 \mu \mathrm{m}$. The upper limits from MSX corresponding to bands C $(12.13 \mu \mathrm{m}), \mathrm{D}(14.65 \mu \mathrm{m})$, and $\mathrm{E}(21.3 \mu \mathrm{m})$ are shown. The solid curve represents the semi-spherical model described in the text. The solid line at FIR wavelengths corresponds to the SED of a central star of $L=1200 L_{\odot}$ and to the $15000 \mathrm{~K}$ surface temperature that is surrounded by an envelope. Possible second blackbody emitter is shown as solid curve at shorter wavelengths. The dashed curve represents a grey body fit of $19 \mathrm{~K}$. The NIR fluxes are explained by scattering (see Sect. 4.3.2).

patchy. It is natural to associate the second blackbody with an accretion disk of about 12 AU radius (outer). Considering the paucity of the data, more refined calculations seem, at present, unwarranted.

The dashed line that fits the long wavelength data in Fig. 5 is a modified Planck curve of the form $v^{2} B_{v}(T)$ with $T=19 \mathrm{~K}$, possibly the emission of the background cloud with a total mass of $350 M_{\odot}$.

\subsubsection{MASS sources}

As shown in Fig. 2, the $\mathrm{CH}_{3} \mathrm{OH}$ peak is offset from the 2MASS source by $\sim 2^{\prime \prime}$. It is highly unlikely that the ATCA observations have errors on the order of $2^{\prime \prime}$ (cf. Sect. 3.1). Though the rms uncertainty in the case of 2MASS data is on the order of $0.4^{\prime \prime}$, we cannot completely rule out that the embedded object triggering maser emission is not associated with the 2MASS source. Because of the high column density towards the centre of the core, it could be that we are seeing light from the embedded object scattered out from the edges of the NIR core. We now discuss such a situation in detail.

The 2MASS $J$ and $H$ bands reveal 3 faint point sources detached from the sub-mm peak, while at $K$, where the resolution is inferior, there is only one single source. These 2MASS points lie far above any possible fits to a spherical cloud model. We first investigated whether they can come from an unrelated foreground star of lower bolometric luminosity but suffering less obscuration. Analysing the observed $J H K$ fluxes in a colour-colour diagram, we found that they cannot be explained by reddening, so we discarded the possibility of a foreground star.

Instead, we propose that we are dealing with three knots of scattered light that escapes from the star into an optically thin

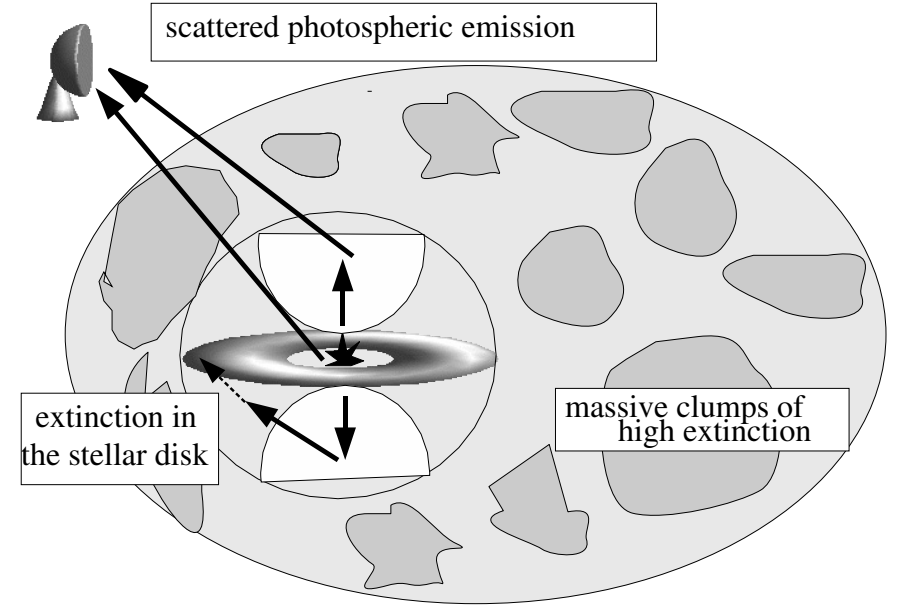

Fig. 6. Illustration of an intuitive model of the source geometry needed to explain the NIR emission. The protostar is part of a massive (several $100 M_{\odot}$ ) clumpy cloud complex. The optically thin outflow cone scatters the stellar light at NIR wavelengths toward the observer. The stellar photons scattered from the cone below the disk is extincted by the optically thick disk; consequently, we observe a uni-polar nebula at NIR.

cone above a hypothetical disk. We estimate the flux scattered by the cone at frequency $v$ from the formula

$S_{v}=\frac{f_{v} L_{v}}{4 \pi D^{2}}$

where $D$ is the source distance and $L_{v}$ the stellar spectral luminosity, so $L=\int L_{v} \mathrm{~d} v$. If $\Omega_{\text {cone }}$ denotes the solid angle of the cone and $\tau_{v}^{\text {sca }}$ its (small) scattering optical depth, then $f_{v}=\tau_{v}^{\mathrm{sca}} \Omega_{\text {cone }} / 4 \pi$. Because of the basically unknown geometry, our estimates are very rough. But assuming the star to be a blackbody, only about one percent of the light has to be scattered (the exact number depends on the stellar temperature).

As regards the wavelength dependence, scattering of pure stellar light would suggest decreasing fluxes at longer (K) wavelengths. However, foreground extinction and, more importantly, hot dust from an accretion disk $(T \sim 1000 \mathrm{~K})$ could easily explain the observed spectral shape at near IR wavelengths. In view of the geometrical requirements posed by the near IR fluxes, we propose a more realistic configuration. In such a model, the protostar is part of a massive (several $100 M_{\odot}$ ) clumpy cloud complex. Such a patchy dust distribution is shown in Fig. 6. As seen in Fig. 2, the emission at NIR is concentrated at only one edge of the BIMA $3 \mathrm{~mm}$ emission. In the configuration envisaged in Fig. 6, the stellar radiation is scattered by the dust clump above the disk at NIR wavelengths toward the observer. However, the stellar photons scattered from regions below the disk suffer severe extinction from the disk and fail to reach the observer at NIR wavelengths, resulting in a uni-polar nebula in NIR.

We therefore regard the $J H K$ knots as further indirect evidence of a stellar disk.

\section{Conclusion}

Our main result is the detection of what is most likely a highmass young stellar object within the IRDC G11.11. If the 
luminosity we derived is an overestimation that corresponds to an intermediate star, this would be the first reported case of CIIMM detection towards an IM mass-star-forming region. This evidence comes from the following observations and arguments:

- $\mathrm{CH}_{3} \mathrm{OH}$ and $\mathrm{H}_{2} \mathrm{O}$ masers appear during the earliest phases of massive star formation, so their detection in G11.11 is evidence of an embedded object.

- The systematic velocity gradient and the linear feature in the $P-V$ diagram of the $\mathrm{CH}_{3} \mathrm{OH}$ lines is a signature of a disk encompassing a protostar.

- The higher excited $\mathrm{NH}_{3}(3,3)$ thermal line points towards a compact and hot central component. The thermal $\mathrm{CH}_{3} \mathrm{OH}$ emission (Leurini et al. in preparation) suggests an outflow, in agreement with the claim by Johnstone et al. (2003), based on $\mathrm{H}_{2} \mathrm{CO}$ obervations. However, Johnstone et al. (2003) do not find any evidence of high-mass star formation.

- The strong FIR fluxes ( $\geq 60$ Jy) derived from analysing the high resolution IRAS data imply a powerful central source (>1000 $\left.L_{\odot}\right)$.

- The radiative transfer additionally requires blackbody emission of about $550 \mathrm{~K}$ from a separate, less embedded source, probably from a disk, in order to reproduce the Spitzer data between 3.6 and $8.0 \mu \mathrm{m}$. A non-spherical geometry is also indicated by the 2MASS NIR fluxes, which can only be explained by scattering and not through reddening.

- The high-extinction estimates also suggest an extremely young object, probably the Class 0 equivalent of a highmass protostar.

Acknowledgements. We thank the ATNF for the observing time allocation at the ATCA. The Australia Telescope is funded by the Commonwealth of Australia for operation as a National Facility managed by CSIRO. We are grateful to M.Voronkov for making our ATCA observations possible. This research has made use of the NASA/ IPAC Infrared Science Archive, which is operated by the Jet Propulsion Laboratory, California Institute of Technology, under contract with the National Aeronautics and Space Administration. T.Pillai was supported for this research through a stipend from the International Max Planck Research School (IMPRS) for Radio and Infrared Astronomy at the University of Bonn. T. Pillai thanks her colleague J. Kauffmann for all the helpful discussions.

\section{Appendix A: Model}

We iteratively solved the energy equation

$$
\int \kappa_{v} J_{v} \mathrm{~d} v=\int \kappa_{v} B_{v}\left(T_{\mathrm{d}}\right) \mathrm{d} v,
$$

together with two integral radiative transfer equations

$$
\begin{aligned}
& I^{+}(\tau)=\mathrm{e}^{-\tau}\left(I^{+}(0)+\int_{0}^{\tau} S(x) \mathrm{e}^{x} \mathrm{~d} x\right) \\
& I^{-}(t)=\mathrm{e}^{-t} \int_{0}^{t} S(x) \mathrm{e}^{x} \mathrm{~d} x .
\end{aligned}
$$

Here $\kappa_{v}$ denotes the dust extinction coefficient, $J_{v}$ the mean intensity, and $B_{v}\left(T_{\mathrm{d}}\right)$ the Planck function at dust temperature $T_{\mathrm{d}}$. In the formulae for the radiative transfer, $I^{+}(\tau)$ is the intensity directed towards the observer at optical depth $\tau$, frequency $v$, and impact parameter $p$, while $I^{-}$refers to beams away from the observer. The source function $S(\tau)$ includes a scattering term. The optical thickness $\tau$ and geometrical length $z$ are related through $\mathrm{d} \tau=\rho \kappa \mathrm{d} z$, where $\rho$ is the density. Index $v$ has been dropped for convenience of writing.

There are two boundary conditions. One states that the standard interstellar radiation field (ISRF) impinges at the cloud surface $\left(I_{v}^{-}=I_{v}^{\mathrm{ISRF}}\right.$ ), the other reads $I^{+}-I^{-}=0$ at $\tau=0$ (where also $z=0)$ at impact parameters $p$ greater than the stellar radius $R_{*}$, and that $I^{+}-I^{-}=F_{*} v / \pi$ at the stellar surface $\left(F_{* v}\right.$ is the stellar flux at frequency $v$ ).

\section{References}

André, P., Ward-Thompson, D., \& Barsony, M. 1993, ApJ, 406, 122 Beuther, H., Walsh, A., Schilke, P., et al. 2002, A\&A, 390, 289

Carey, S. J., Clark, F. O., Egan, M. P., et al. 1998, ApJ, 508, 721

Carey, S. J., Feldman, P. A., Redman, R. O., et al. 2000, ApJ, 543, L157

Cesaroni, R. 1990, A\&A, 233, 513

Egan, M. P., Shipman, R. F., Price, S. D., et al. 1998, ApJ, 494, L199 Elitzur, M., Hollenbach, D. J., \& McKee, C. F. 1989, ApJ, 346, 983

Elmegreen, B. G., \& Morris, M. 1979, ApJ, 229, 593

Garay, G., Brooks, K. J., Mardones, D., Norris, R. P., \& Burton, M. G. 2002, ApJ, 579, 678

Garay, G., \& Lizano, S. 1999, PASP, 111, 1049

Hill, T., Burton, M. G., Minier, V., et al. 2005, MNRAS, 796

Johnstone, D., Fiege, J. D., Redman, R. O., Feldman, P. A., \& Carey, S. J. 2003, ApJ, 588, L37

Krügel, E. 2003, Astron. Geophys., 44, 35

Kurtz, S. 2002, in Hot Star Workshop III: The Earliest Phases of Massive Star Birth, ASP Conf. Ser., 267, 81

Minier, V., Booth, R. S., \& Conway, J. E. 1998, A\&A, 336, L5

Minier, V., Ellingsen, S. P., Norris, R. P., \& Booth, R. S. 2003, A\&A, 403, 1095

Motte, F., Andre, P., \& Neri, R. 1998, A\&A, 336, 150

Motte, F., Schilke, P., \& Lis, D. C. 2003, ApJ, 582, 277

Norris, R. P., Byleveld, S. E., Diamond, P. J., et al. 1998, ApJ, 508, 275

Perault, M., Omont, A., Simon, G., et al. 1996, A\&A, 315, L165

Phillips, C. J., Norris, R. P., Ellingsen, S. P., \& McCulloch, P. M. 1998, MNRAS, 300, 1131

Ponomarev, V. O., Smith, H. A., \& Strelnitski, V. S. 1994, ApJ, 424, 976

Redman, R. O., Feldman, P. A., Wyrowski, F., et al. 2003, ApJ, 586, 1127

Reid, M. J., Schneps, M. H., Moran, J. M., et al. 1988, ApJ, 330, 809

Sandell, G. 2000, A\&A, 358, 242

Teyssier, D., Hennebelle, P., \& Pérault, M. 2002, A\&A, 382, 624

Walsh, A. J., Burton, M. G., Hyland, A. R., \& Robinson, G. 1998, MNRAS, 301, 640

Walsh, A. J., Bertoldi, F., Burton, M. G., \& Nikola, T. 2001, MNRAS, 326,36

Walsh, A. J., Macdonald, G. H., Alvey, N. D. S., Burton, M. G., \& Lee, J.-K. 2003, A\&A, 410, 597

Wyrowski, F., Schilke, P., Thorwirth, S., Menten, K. M., \& Winnewisser, G. 2003, ApJ, 586, 344 